Original paper

\title{
High velocity pulse biopsy device enables controllable and precise needle insertion and high yield tissue acquisition
}

\author{
Kai-Uwe Schässburger ${ }^{\mathrm{a}, *}$, Stefan Paepke ${ }^{\mathrm{b}}$, Ariel Saracco ${ }^{\mathrm{c}}$, Edward Azavedo ${ }^{\mathrm{d}}$, Christina Ekström ${ }^{\mathrm{e}}$, \\ Hans Wiksell ${ }^{\mathrm{a}}$ \\ ${ }^{a}$ Department of Molecular Medicine and Surgery, Karolinska Institutet, Stockholm, Sweden \\ ${ }^{\mathrm{b}}$ Interdisciplinary Breast Centre, Klinikum rechts der Isar der TU München, München, Germany \\ ${ }^{\mathrm{c}}$ Department of Diagnostic Radiology, Division of Breast Imaging, Södersjukhuset, Stockholm, Sweden \\ d Department of Diagnostic Radiology, Karolinska University Hospital, Stockholm, Sweden \\ e Department of Pathology, Unilabs AB, Capio S:t Görans Hospital, Stockholm, Sweden
}

\section{A R T I C L E I N F O}

\section{Keywords:}

Breast

Axilla

Biopsy

CNB

$\mathrm{VAB}$

Needle

Ultrasound

\begin{abstract}
A B S T R A C T
Minimally invasive biopsies are a cornerstone of breast cancer management with ultrasound being the preferred guidance modality. New developments in breast cancer management and advances in imaging technologies bring new challenges to current biopsy methodologies. A new biopsy device (NeoNavia ${ }^{\circledR}$ biopsy system, 14 G) was developed. It incorporates a pneumatic needle insertion mechanism that is intended to provide better control of needle progression and enable stepwise insertion without noticeable deformation or displacement of surrounding tissue as visualized under ultrasound. A new method of tissue acquisition was designed to achieve a sampling yield higher than standard methodologies. Needle dynamics was assessed on a specifically designed test bed and sampling performance was compared to a Magnum ${ }^{\circledR}$ biopsy instrument (Bard, Covington, GA, USA) in representative tissue models. The histological quality of samples obtained ex-vivo was evaluated. A pneumatic pulse was measured to accelerate the needle to a maximum velocity of $21.2 \pm 2.5 \mathrm{~m} / \mathrm{s}$ on a stroke length of $2.5 \mathrm{~mm}$, achieving significantly higher acceleration, maximum velocity and power than current biopsy devices. Mean weight of samples obtained by the NeoNavia device were 3.5, 4.6, and 4.3 times higher when sampling was performed in turkey breast, calf thymus and swine pancreas, respectively, as compared to samples obtained with the Magnum instrument. Ex-vivo analysis indicates that the method of tissue acquisition has no apparent negative impact on the histopathologic quality of obtained samples.
\end{abstract}

\section{Introduction}

Minimally invasive biopsy is a well-established method of obtaining samples from breast tissue that demonstrates suspicious lesions on imaging. As many as 1.7 million such procedures are performed each year in the US alone [1]. Minimally invasive biopsy of breast and axillary lymph nodes enables assessment of tumor biology and staging for an individual treatment of breast cancer patients [2,3]. Various studies have substantiated the diagnostic value of core needle biopsy (CNB) and vacuum-assisted biopsy (VAB) procedures [4] which have become the gold standard method for the initial assessment of suspicious breast lesions [5]. For minimally invasive biopsies of lesions the preferred guidance modality is ultrasound (US) as it offers real time visualization, ready access to all parts of the breast including the axilla and, compared to modalities such as mammography and magnetic resonance imaging (MRI), a shorter procedure time, increased patient comfort and no exposure to ionizing radiation or intravenous contrast media [6].

Core needle biopsy devices currently used in breast diagnostics were conceived in the late 80's and are based on a tissue cutting or punching mechanism. The insertion of the CNB needle into the tumor is usually powered by a spring-loaded mechanism which thrusts the needle into the tumor. The outer diameter of a CNB needle is in the range of 18-14 G (1.3-2.1 mm). Vacuum assisted biopsy devices were first presented in the mid-90's. These devices are based on CNB technique, but additionally employ a vacuum to prolapse the tissue into the aperture while also incorporating larger needle diameters in the range of 7-14 G (4.6-2.1 mm). Illustrations of $\mathrm{CNB}$ and $\mathrm{VAB}$ mechanisms are given in Fig. 1. Both $\mathrm{CNB}$ and VAB incorporate needle placement mechanisms with predetermined stroke lengths, can incorporate large and sharp needle tips as well as needle designs that only use a fraction of the biopsy needle volume for actual sample acquisition. Challenging cases of ultrasound-guided breast biopsies described in the literature include

\footnotetext{
* Corresponding author at: Department of Molecular Medicine and Surgery, Karolinska Institutet, NeoDynamics AB, Lejonvägen 14, 181 32 Lidingö, Sweden.

E-mail address: kai-uwe.schassburger@ki.se (K.-U. Schässburger).
} 


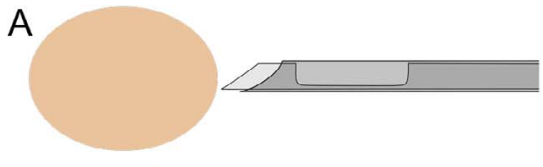

B
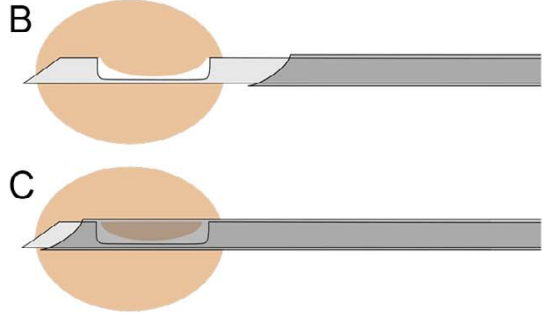
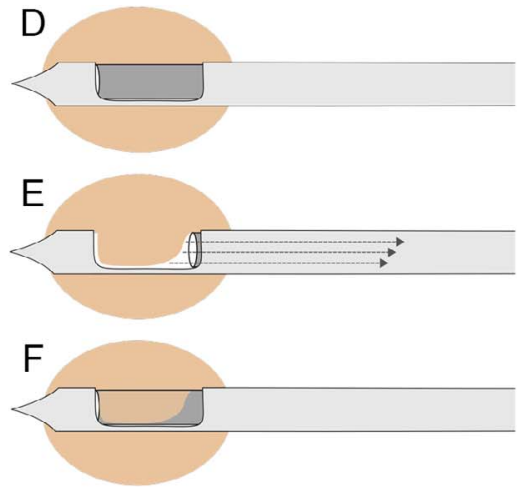

Fig. 1. Illustration of insertion and sampling technique of CNB and VAB in the left and right column, respectively. (A) The inner and outer cannula of the CNB are aligned as the needle is inserted through healthy tissue towards the suspicious lesion. The aperture is closed. (B) The inner needle incorporating a needle tip of around $5 \mathrm{~mm}$ is fired into the lesion over a distance of approximately $20 \mathrm{~mm}$. (C) The firing of the inner needle automatically triggers a system for immediate advancement of the outer cannula to cut the sampled tissue trapped inside the aperture. The biopsy needle is subsequently withdrawn from the patient and the outer cannula is retracted to expose the sample inside the notch. (D) The VAB needle is inserted to the area of interest with the aperture closed by the inner cutting cannula. Placement is achieved either by manually pushing the needle forward or via a placement mechanism. VAB needles incorporate needle tips of around $10 \mathrm{~mm}$ (E) Once at the

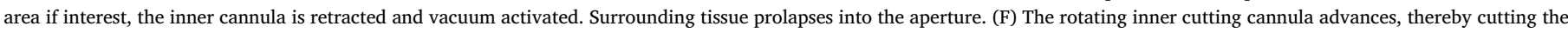

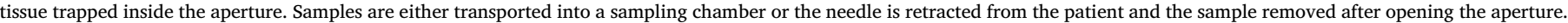

small breast lesions, tumors near the skin surface, deep lesions, lesions adjacent to silicon implants and those near the chest wall, calcified lesions and mobile lesions such as small fibroadenoma [7]. Biopsies in the axilla, with its specific topography, i.e. the closeness of the lymph nodes to blood vessels and nerves, also poses specific challenges, limiting the practicability of current biopsy devices [8]. It is reported that needle insertion towards the lesion may be cumbersome in patients with dense breasts or fibrosis [7]. Challenging cases are generally those that present with a lesion that is hard to reach or target, cases where the lesion gets pushed aside by the biopsy needle tip resulting in the collection of non-representative samples and lesions in the vicinity of delicate anatomical structures.

The sensitivity and specificity of high resolution ultrasound and associated technologies, such as elastography and optoacoustic imaging, are continually improving with the result of increasing diagnostic potential. The combination of increasingly sensitive imaging techniques and expanding breast cancer screening programs, early detection in high risk patient groups and increased use of breast sonography in daily care has seen an increase in the reporting of small and unclear findings, where histological clarification is indicated but current biopsy methods reach their limits $[7,9]$. As with imaging technology, the management of breast cancer is constantly evolving and with these changes come new challenges to existing biopsy methodologies. The need for axillary lymph node biopsies has increased with the growing number of patients receiving neoadjuvant chemotherapy. Current developments include the analysis of phenotypic and genetic intratumoral heterogeneity [10], an emerging systematic approach to biopsies of distant metastasis for improved individualization of therapy [11] and the use of image-guided biopsies to diagnose a pathological complete response in the breast after neoadjuvant chemotherapy.

Some of the current challenges faced by existing biopsy techniques could be overcome by finer needle control during insertion and improved tissue sampling efficiency. Characteristics of needle insertion are determined by the mechanics of tissue-cutting during needle penetration. Applying the framework of fracture mechanics, it has previously been proposed and validated that the tissue-cutting process during needle insertion can be split into two distinct phases [12]. In the first phase, tissue deflects progressively as the needle force builds up to the amount required to initiate cutting. The second phase occurs once the tissue has fractured and is characterized by the force subsequently stabilizing or decreasing. Increased needle acceleration minimizes tissue deflection in the first phase by creating a high force leading to instant tissue fracture. Increased needle velocity minimizes the cutting force needed for tissue fracture and minimizes tissue displacement during the second phase. As proposed in earlier work by Wiksell et al. [13], high needle acceleration and velocity can be achieved by applying pneumatic pulses to the biopsy needle. In combination with a short stroke length, in the order of millimeters, and the ability to apply multiple consecutive pulses it might be expected to enable the physician to deploy the biopsy needle into the breast in a stepwise and controlled manner without noticeable deformation or displacement of surrounding tissue on the ultrasound image. The sampling needle should further use a needle design where the complete needle volume can be used for tissue acquisition.

We report here on the development and preclinical validation of a high velocity pulsed-insertion biopsy device that incorporates a pneumatic insertion mechanism and novel sampling needle design. We describe needle dynamics using a specially designed needle trajectory test bed, compared its sampling performance with a routinely used CNB device on representative bench models and assessed the histological quality of samples collected in an ex-vivo setting.

\section{Materials and methods}

\subsection{NeoNavia biopsy system}

\subsubsection{Pneumatic needle insertion mechanism}

Building on previous institutional work reported by Wiksell et al. [13], the developed insertion mechanism reported here is based on a pneumatically driven reciprocating stainless steel weight that transfers energy via a piston to the biopsy needle (see Fig. 2). The design incorporates a $7 \mathrm{~cm}$ stainless steel tube containing a loose steel projectile that weighs $12 \mathrm{~g}$. Magnets hold the projectile in place at the proximal end of the steel tube. A polyvinyl chloride (PVC) tube is attached to the proximal end that is connected to a regulator and compressor generating pressurized air at 4.5 bar gauge pressure. As a high-speed response solenoid valve is opened for $50 \mathrm{~ms}$, pressure behind the projectile builds up until it overcomes the magnetic force holding it back.

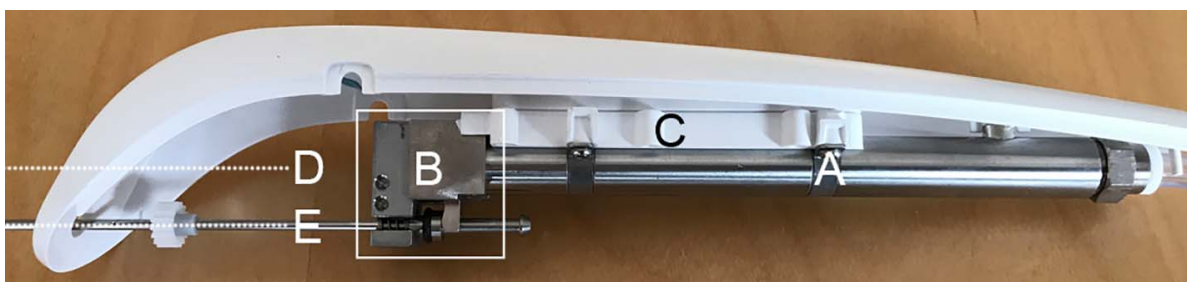

Fig. 2. Needle insertion mechanism. The steel tube incorporating the projectile is shown (A) as well as the piston arrangement (B). (C) The chamber that contains the pressure which will return the projectile to its initial position. The offset axis of the projectile trajectory and the biopsy needle are depicted with dashed lines (D) and (E), respectively. 
At this point the projectile is propelled forward through the steel tube. A piston arrangement at the distal end of the tube is used to transfer the energy from the accelerated projectile to the biopsy needle. Pressure builds up inside a compartment at the distal end of the steel tube as the projectile travels forward. This pressure subsequently returns the projectile to its starting position. The biopsy needle movement is restricted to allow a maximum stroke length of around $2.5 \mathrm{~mm}$ before it is stopped by a rubber ring and returned to its original position by a coil spring. The axis of the steel tube and the sampling needle are offset to each other. This enables for a solid trocar to be inserted into, and withdrawn from the sampling needle via its proximal end. When this insertion mechanism is integrated into a biopsy hand piece the movement of the needle created by the insertion mechanism in combination with manual advancement of the biopsy hand piece generates a stepwise needle advancement. The needle is, in effect, pulsing into the tissue. More details on the mechanics are given elsewhere [14].

\subsubsection{Sampling needle design}

To enable for a maximum sampling yield with minimal tissue trauma a distal-tip sampling needle design is employed. A distal-tip sampling needle is essentially a hollow needle with an open needle tip. The complete inner needle volume can be used as a sampling cavity without the need for a solid and sharp needle tip. A sharpening grind was applied to the inside of the distal needle tip, thereby maximizing the tissue volume that can enter into the needle (see Fig. 3). Negative pressure is applied to the sampling needle, known to increase the sampling yield of distal-tip biopsy needles. The negative pressure counters the friction experienced by the tissue due to the internal needle wall during sampling. As the needle cuts into the tissue the sample is pulled into the needle by the vacuum [15] which is transferred to the needle via a number of small holes in the needle wall at the proximal end and a suction connector (see Fig. 3). The suction connector is connected to a compressor that is capable of applying a
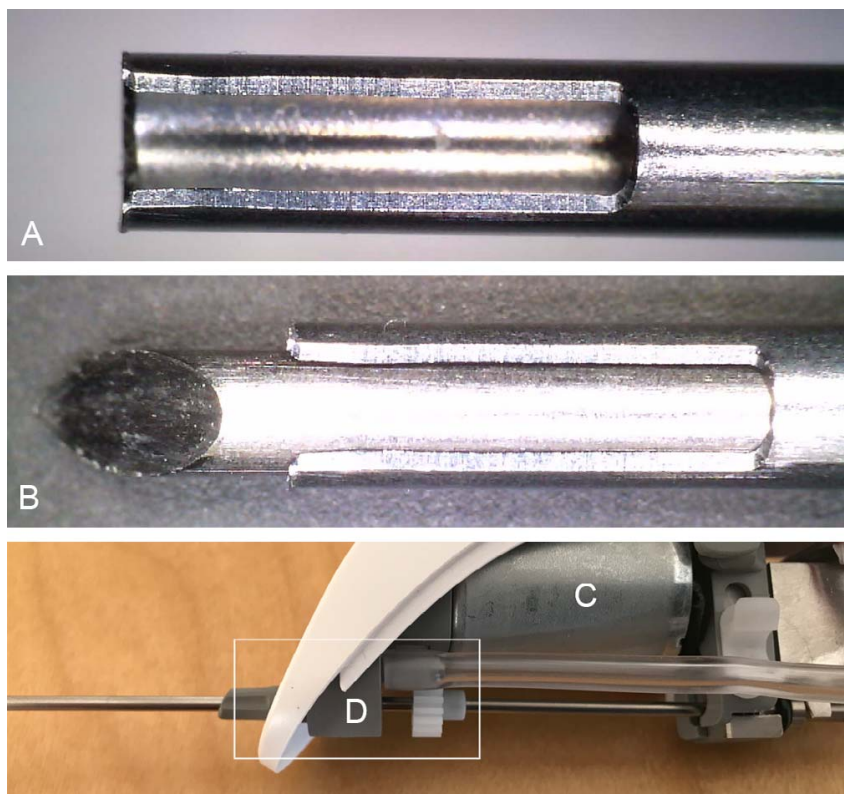

Fig. 3. Magnified $(35 \times)$ pictures of the distal tip of the biopsy needle and tissue acquisition mechanism. (A) shows the biopsy needle when it is inserted into the lesion. The trocar is at a position $60 \mathrm{~mm}$ retracted into the sampling needle thereby exposing the distal tip of the sampling needle and creating a sampling cavity of corresponding length. The slit can be seen as well as the inward grind at the needle tip. (B) The distal part of the biopsy needle with the trocar in an extended position. The trocar prevents healthy tissue from entering the sampling needle during insertion towards the lesion. (C) The electrical motor which rotates the needle via cogwheels to separate the sample from surrounding tissue. (D) The suction connector that allows for longitudinal and rotational needle movement.
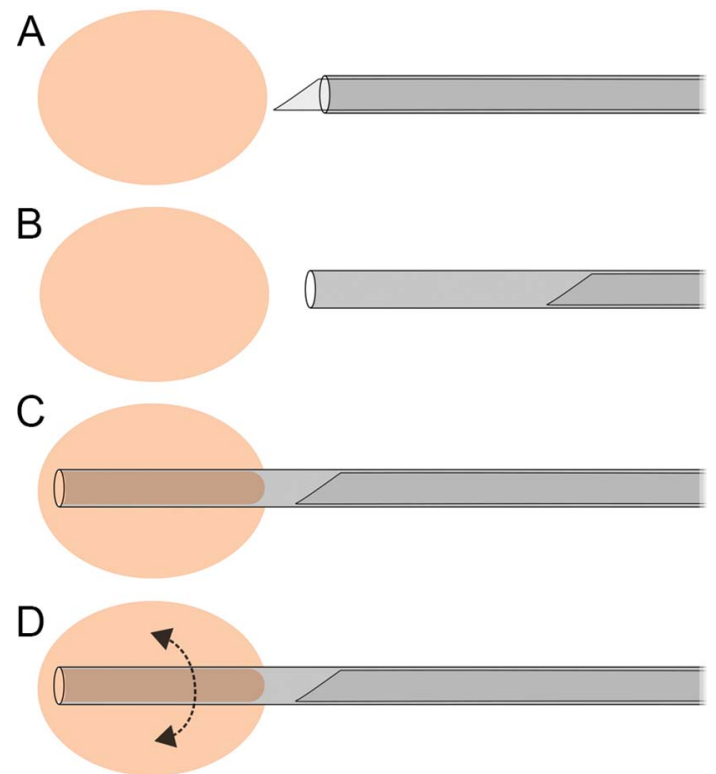

E



Fig. 4. Illustration of NeoNavia sampling methodology. (A) The needle is advanced though healthy tissue towards the suspicious lesion with the trocar extended. (B) With the tip of the trocar put at the suspicious lesion, the trocar is retracted and exposes the cavity of the distal tip sampling needle. (C) Pneumatic pulses are used to insert the sampling needle into the lesion. (D) When the needle has been inserted a desired length it is rotated to cut off the sample. (E) The needle is subsequently retracted from the patient and the trocar is extended to eject the tissue sample at the distal tip of the biopsy needle.

negative pressure of $60 \mathrm{kPa}$ inside the sampling needle within $5 \mathrm{~s}$.

Once in the needle bore that sample has to be cut off and separated from surrounding tissue. Rotating the needle rapidly once it has entered the tissue serves to cut the sample from surrounding tissue, which is applied by clockwise needle turning for $50 \mathrm{~ms}$ followed by $50 \mathrm{~ms}$ pause and a subsequent $50 \mathrm{~ms}$ of counter clockwise turn. Momentum applied to the tissue is increased by means of a slit incorporated in the body of the needle (see Fig. 3). The rotational movement is generated by an electric motor and transferred to the needle via cogwheels (see Fig. 3). The cogwheels permit for longitudinal needle movement during application of pneumatic pulses whereas the suction connector permits both longitudinal and rotational needle movement. Additional details of the mechanics behind the sampling mechanism are provided elsewhere [16].

\subsubsection{Biopsy procedure}

An illustration of a biopsy procedure using the developed device is provided in Fig. 4. The needle is initially advanced though healthy tissue towards the suspicious lesion with the trocar extended, thereby blocking healthy tissue from entering the needle (see Fig. 3). Where manual penetration of the tissue is problematic in case of e.g. dense breast tissue or when increased precision is necessary, pneumatic pulses are applied. When the tip of the trocar has reached the suspicious lesion, the trocar is retracted exposing the cavity of the distal tip sampling needle. Pneumatic pulses are used to insert the sampling needle into the lesion with vacuum suction aiding in filling the needle with tissue during advancement. The sampling needle can be inserted stepwise into the lesion to collect samples of corresponding length. Maximum insertion length is restricted to $6 \mathrm{~cm}$. Once the desired insertion length has been achieved the needle is rotated to cut off the sample. The sampling needle is retracted from the patient and the trocar extended to eject the tissue sample from the distal tip of the needle. 


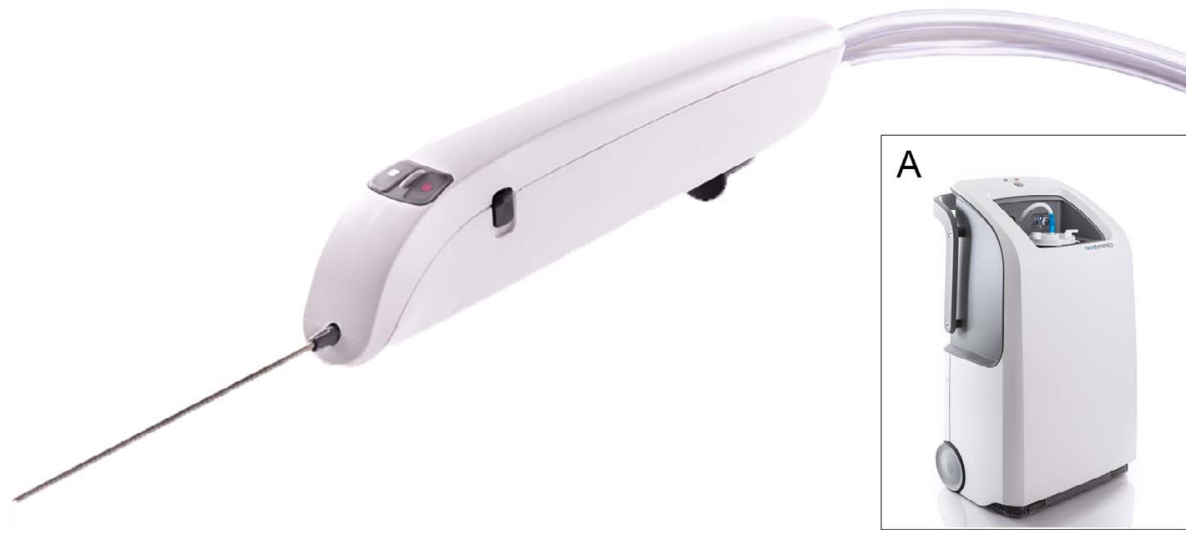

Fig. 5. Image of the NeoNavia biopsy device and base unit (A). The lever on the bottom of the device is used to extend the trocar and fixate it to the sampling needle with an internal spring mechanism. This is done when preparing the device for sampling to enable a sharp dissection tip while blocking the needle tip to keep healthy tissue from entering the sampling needle. The trocar retraction buttons on each side of the handle are used to retract the trocar, which allows sampling to start by unblocking the needle tip and initiating vacuum suction capability through the sampling needle. When the operator pushes the pulse button on top of the device a pneumatic pulse is activated which results in a forward movement of the biopsy needle. After filling the sampling needle with an adequate amount of tissue by moving the sampling needle through the suspicious lesion using pneumatic pulses, the sample is cut using the cutting button. Pushing the button initiates a rotational motion that separates the tissue inside the sampling needle from sur-

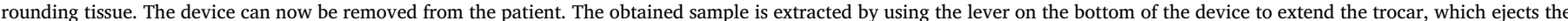

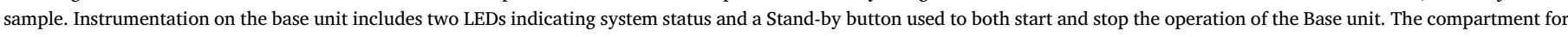
the vacuum canister and connections for the biopsy device can be seen. The vacuum canister protects the compressor from contamination.

\subsubsection{System integration}

The needle insertion mechanism and sampling needle design were incorporated into a new biopsy system. Taking into account user aspects regarding cable length, start time, noise levels, size requirements, for example, and in compliance with regulatory requirements on safety and efficacy the NeoNavia biopsy system (NeoDynamics, Sweden) was developed and a CE-marking obtained. It is a tethered system with a base unit supplying the biopsy device with power and controlling its operation during the sampling procedure (Fig. 5). The NeoNavia biopsy device is sterile and intended for single-use only. It consists of a conical handle that incorporates the pneumatic driver and a $14 \mathrm{G}$ distal-tip sampling needle with an integrated trocar. The base unit is a floor positioned mobile base unit powered from a standard mains power outlet. The exterior of the base unit includes a ventilation outlet and intake enabling air-cooling of the system during operation. Key components of the base unit are two oil-free piston compressors. One compressor creates pressurized air for the pneumatic driver and the other provides suction for the sampling needle. The compressors are mounted together with a fan and additional pneumatic components to safely control their operation inside a sound box to minimize noise.

\subsection{Needle trajectory test bed}

The custom made test bed that was developed to measure needle velocity consists of a fixture for the hand piece and a sensor unit mounted on a stable base plate (Fig. 6) as well as a personal computer (PC) with a data acquisition (DAQ) device for controlling the test hardware and sampling data. The sensor unit incorporates a linear image sensor, two light emitting diodes and a stepper motor. The complementary metal-oxide semiconductor (CMOS) sensor (S11105, Hamamatsu Photonics) incorporates 512 pixels arranged linearly in the space of $6.4 \mathrm{~mm}$ (Fig. 6) and provides a maximum video data rate of $50 \mathrm{MHz}$. The LED has a spread angle of 1 degree and delivers a power of $1 \mathrm{~W}$. The stepper motor is used to control the longitudinal position of the sensor unit. The PC (Intel i7, 12 Gb RAM running Microsoft Windows, version 7) incorporates a digital input/output device (PCIe6537B, National Instruments) that features a maximum clock rate of $50 \mathrm{MHz}$ and receives the video data from the sensor. The PC is connected to the DAQ device (USB-6212, National Instruments) that is used to control the stepper motor and triggers the opening of the pressure valve inside the base unit. The software run by the PC was developed in LabView 2012 (National Instruments). It allows the operator to set the sampling window and control activation of the pressure pulse. Resulting raw data (needle position and time) can be imported to spreadsheet software. A single frame for signals from 512 channels takes up 600 bits which results in a maximum frame rate of $83.3 \mathrm{kHz}$ at a $50 \mathrm{MHz}$ clock rate. Needle trajectory was measured for 1.5, 2.5, 3.5, and 4.5 bar gauge pressure. Ten measurement series were performed for every pressure setting.

\subsection{Sampling yield testing in tissue models}

Testing was performed in turkey breast, swine pancreas and calf thymus. A custom-made test box was manufactured from transparent plastic and equipped with holes in the wall to allow for sampling through the side wall. A $280 \mathrm{~g}$ lid was placed on the tissue model to hold it in place. The NeoNavia biopsy device ( $14 \mathrm{G}$ needle) was compared with a Magnum reusable core biopsy instrument (Bard, Covington, GA, USA), also equipped with a $14 \mathrm{G}$ needle employing a stroke length of $22 \mathrm{~mm}$. The Magnum device employs the mechanism depicted in Fig. 1 and was judged to be a suitable benchmark device since it was the first on the market and is today one of the most widely used CNB devices in clinical practice. In order to generate comparable data, the NeoNavia biopsy needle was inserted $22 \mathrm{~mm}$ into the tissue, corresponding to the stroke length of the comparison device. Obtained tissue samples were weighed using a precision balance (Sartorius CD64 scale; 0-64 g, resolution $\pm 0.1 \mathrm{mg}$ ). Since fluid content in all obtained samples could influence results, the samples were placed on an absorbent paper towel so that any excess fluid would be absorbed and subsequently placed on the scales with tweezers. All procedures were performed with the tissue at room temperature.

\subsection{Pathologic analysis of tissue samples from resected specimen}

Ethical permits (Dnr. 2013/705-31/2; 2014/824-32) were obtained for ex-vivo testing at Unilabs $\mathrm{AB}$ pathology department at Capio S:t Görans Sjukhus, Stockholm (Sweden). Patients with a biopsy-proven breast cancer $\geq 15 \mathrm{~mm}$ and planned for surgical excision were recruited to the study. After surgery to remove the lesion, specimens were transported immediately to the pathology department at Capio S:t Görans Sjukhus. The resected specimen was cut in the plane of the tumor center. Samples were subsequently obtained using the NeoNavia Biopsy System by technical personnel experienced in its use. After routine histopathological preparation tissue samples were analyzed by a senior pathologist (CE) with 14 years experience of breast pathology. The aim was to review whether or not the histological integrity of the tissue sample was disturbed in any way by the rotational movement of the needle during sampling or by the trocar ejecting the tissue from the sampling needle. The analysis reviewed how sample quality compared with samples collected in routine clinical testing. Six specimens were sampled with configurations of needle design and rotation characteristics that were later discarded due to issues of technical performance 


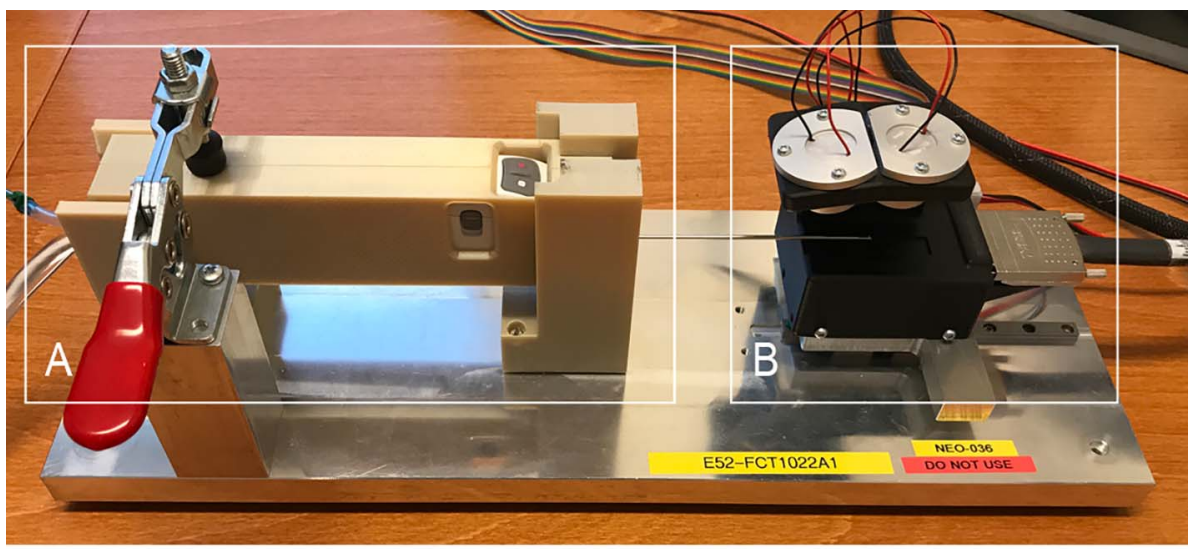

Fig. 6. Images of the velocity test bed. (A) The fixture for the hand piece and (B) the movable sensor unit. (C) A closeup of the sensor unit with a biopsy needle to its left. As the needle tip is pulsing forward it shadows the light from the LED from reaching the sensors.

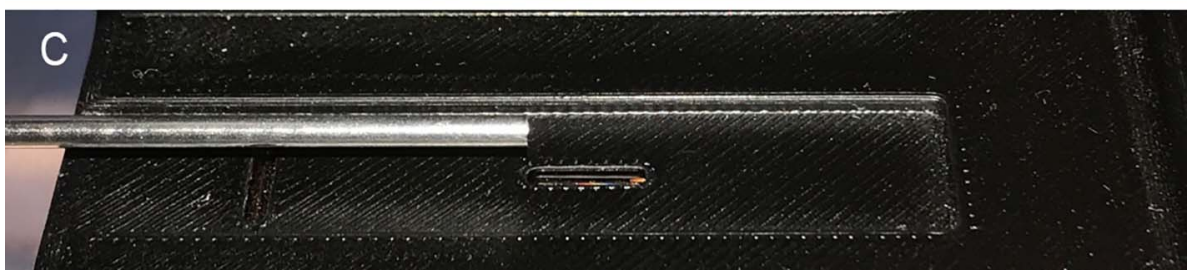

and so were excluded from this analysis. The NeoNavia Biopsy System with sampling ability characteristics (needle design, mechanical rotation pattern, micro-pulse characteristics and vacuum level) as evaluated in this work was used in 11 specimens.

\subsection{Statistical analysis}

For comparing the sampling yield between the NeoNavia biopsy system and the Magnum device an independent samples $t$-test was performed using SPSS statistics Version 24 (IBM, USA).

\section{Results}

\subsection{Needle velocity}

Fig. 7 shows the result of needle trajectory measurements. The slope of the curves, and therefore velocity of the needle, is largest during the first trajectory ramp. For the four different pressure settings the average maximum velocities \pm standard deviations (SD) were $11.6 \pm 1.2 \mathrm{~m} / \mathrm{s}$, $15.8 \pm 1.6 \mathrm{~m} / \mathrm{s}, 19.5 \pm 1.8 \mathrm{~m} / \mathrm{s}$ and $21.2 \pm 2.5 \mathrm{~m} / \mathrm{s}$, respectively. In all cases these velocities was reached well within the first millimeter of biopsy needle progression.

\subsection{Sampling yield in tissue models}

In all three tissue models the NeoNavia biopsy device delivered

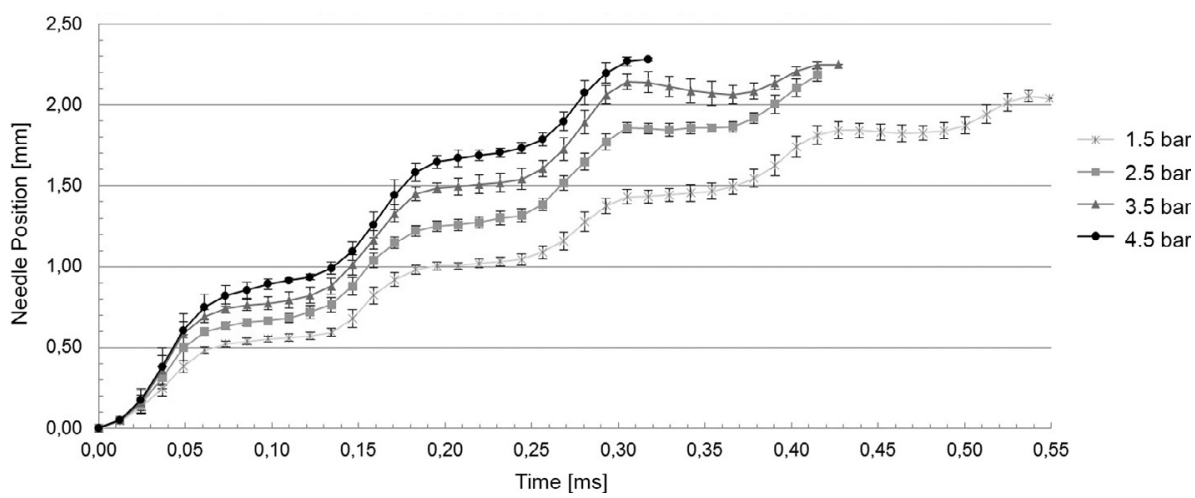

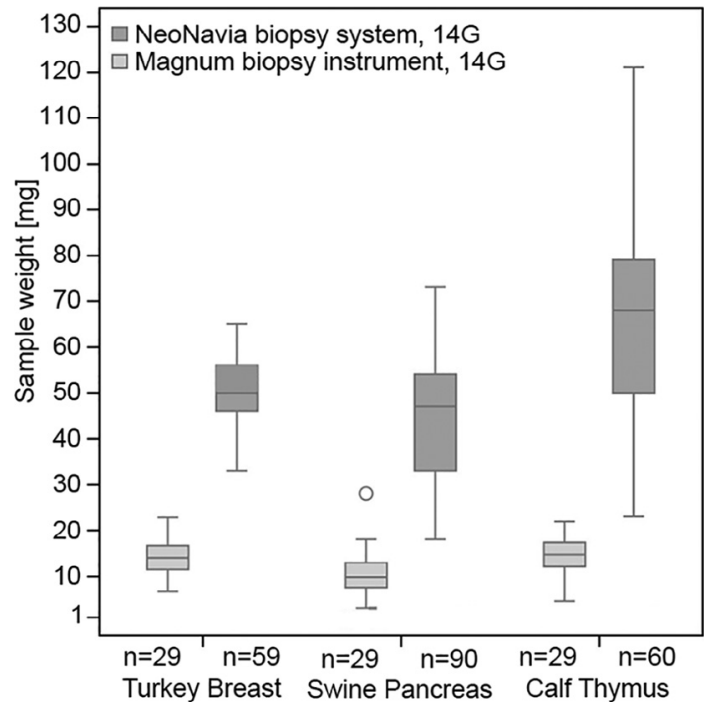

Fig. 8. Box-plot showing sampling yield for the NeoNavia biopsy system and a comparative CNB device in three different tissue models.

heavier samples than the Magnum instrument with identical needle diameter and insertion length into the tissue model (see Fig. 8). In turkey breast, mean sample weight \pm SD for the NeoNavia and

Fig. 7. Plot showing the needle trajectory for four different pressure settings. Trajectories show the mean position values and standard deviations of 10 measurements series for respective pressure setting. Every series was cut off at the point of furthest needle extension. 
Magnum were $49.6 \pm 8.0 \mathrm{mg}$ and $14.3 \pm 3.6 \mathrm{mg}$, respectively, with the difference demonstrating statistical significance $(\mathrm{t}(33,63)=22.56$, $\mathrm{p}<.01$ ). In swine pancreas, delivered samples had a mean weight \pm SD of $45.8 \pm 15.0 \mathrm{mg}$ and $10.1 \pm 3.8 \mathrm{mg}$ for the NeoNavia and Magnum respectively with the difference achieving statistical significance $(\mathrm{t}(29.19)=11.99, \mathrm{p}<.01)$. Finally, in calf thymus tissue the obtained samples had a mean weight \pm SD of $66.5 \pm 14.3 \mathrm{mg}$ and $14.3 \pm 4.0 \mathrm{mg}$ for the NeoNavia and Magnum, respectively. The difference was statistically significant $(\mathrm{t}(28.72)=11.30, \mathrm{p}<.01)$.

\subsection{Pathologic specimen sampling}

Thirty-eight samples were obtained from 11 specimens using the NeoNavia biopsy device. As judged by the pathologist, the quality of tissue samples delivered was equivalent to the quality of tissue samples obtained with standard CNB and VAB devices used in current clinical practice. The severity of damage present in the tissue samples (showing in the form of crush artefacts or sample fragmentation) delivered by the NeoNavia biopsy device was also equivalent to the level of damage present in samples obtained with standard devices in current clinical practice as judged by the pathologist. For pictures of the sampling procedure and examples of samples see Fig. 9.

\section{Discussion}

We present a newly developed biopsy system for breast cancer that incorporates a pneumatic needle insertion mechanism and a new method of sample acquisition.

The maximum sampling needle velocity of the NeoNavia biopsy device in air was $21.2 \mathrm{~m} / \mathrm{s}$ on a stroke length of approximately $2.5 \mathrm{~mm}$. In comparison, maximum velocities in air have been reported to be $8 \mathrm{~m} / \mathrm{s}$ for the Magnum and 10-21 m/s for four disposable CNB devices [17]. These devices reached their respective maximum velocities at the end of the spring accelerated travel distance of around $20 \mathrm{~mm}$. The velocity of the sampling needle employed in the NeoNavia biopsy device depends on the pressure in the pneumatic driver. The specific pressure selected for use in the NeoNavia biopsy system was chosen following empirical testing in different tissue models that confirmed a pressure level necessary to enable inertia stabilization of surrounding tissue, i.e. the sampling needle pulses forward with minimal movement of surrounding tissue. The potential of spring loaded needles to penetrate tissue is determined by their maximum kinetic energy [18]. The higher the energy, the higher the force that can be applied by the needle as it passes through the lesion. The ballistic characteristics of the pneumatically driven sampling needle are fundamentally different from those of needles propelled by a spring-loaded mechanism. As the pneumatically driven insertion mechanism accelerates the sampling needle significantly faster, we include acceleration and power as part of our analysis. Table 1 shows a direct comparison with CNB devices that have been studied in detail by other authors. The pneumatic driver achieves a higher maximum needle velocity with a significantly higher acceleration $(x>200)$ and more power $(x>150)$ being delivered to the sampling needle than seen in CNB devices. The higher acceleration and power sees stabilization of surrounding tissue during needle insertion. The biopsy needle trajectory of the NeoNavia biopsy device is characterized by an oscillating progression which can be explained by the stress wave created by the elastic impact of the projectile resulting in elastic deformations of the needle as it propagates through the body of the needle. The velocity measured was highest during the first trajectory ramp. This may be a consequence of friction, the return spring acting on the needle as well as energy lost to surrounding material causing the stress amplitude to decrease and thereby slowing down the needle tip. The observed dynamic behavior of the needle could be further explored using, for example, finite element method simulation as well as test series in tissue models.

In all tested tissue models the new needle design and method of tissue acquisition of the NeoNavia biopsy device achieved larger volume of samples than the Magnum device. Since both tested devices employed identical needle diameter and insertion length the findings imply that the NeoNavia biopsy device provides better tissue yield which could have positive results in the clinical setting such as fewer needle insertions needed for achieving a conclusive diagnosis. Increase in tissue yield was more pronounced in swine pancreas and calf thymes than in turkey breast suggesting that the effect of applied negative pressure within the needle is greater for softer tissue textures. The data also indicates that the sampling mechanism produces more disperse results in these tissues. Since turkey breast is the most widely used model in the literature, results from other published reports were compared and are shown in Fig. 10. Results from our test series on the Magnum instrument correlate with previous reported data from identical or technically equivalent devices such as the Monopty and ProMag 2.2 [19]. In general, the NeoNavia biopsy device delivered significantly more tissue than $14 \mathrm{G}$ CNB devices, provides tissue amounts comparable to $14 \mathrm{G} \mathrm{VAB}$ devices, though less than $\mathrm{VAB}$ devices equipped with significantly larger needle diameters. The comparison considers neither the fact that VAB devices can deliver multiple samples with a single insertion, nor the ability of the NeoNavia biopsy device to obtain longer samples than CNB and VAB devices. Furthermore, direct comparisons to VAB devices can be difficult since they employ needles with a non-circular cross section due to a canal located beneath the sampling chamber. Finally, although the bench models covered a wide range of tissue structures, they do not fully represent the texture and structure of in-vivo breast and axillary tissue.

Results from ex-vivo analysis show that there is no apparent negative impact on the pathologic quality of the obtained tissue samples by rotation or subsequent ejection of the samples. Any fundamental negative impact would have been detected by our test series. Further work is needed to analyze quantitatively whether samples are superior in quality or yield to samples currently obtained in clinical practice and to compare potential damage to surrounding tissue during needle insertion.

The data presented here indicates that the pneumatic insertion mechanism enables for a novel way of needle insertion into the breast and axilla. The developed method of sample acquisition delivers a high tissue yield. Clinical studies will need to demonstrate to what extent and in which specific lesions these characteristics can transform into clinically relevant benefits such as better diagnostic accuracy, shorter procedure time or less patient trauma when compared to existing
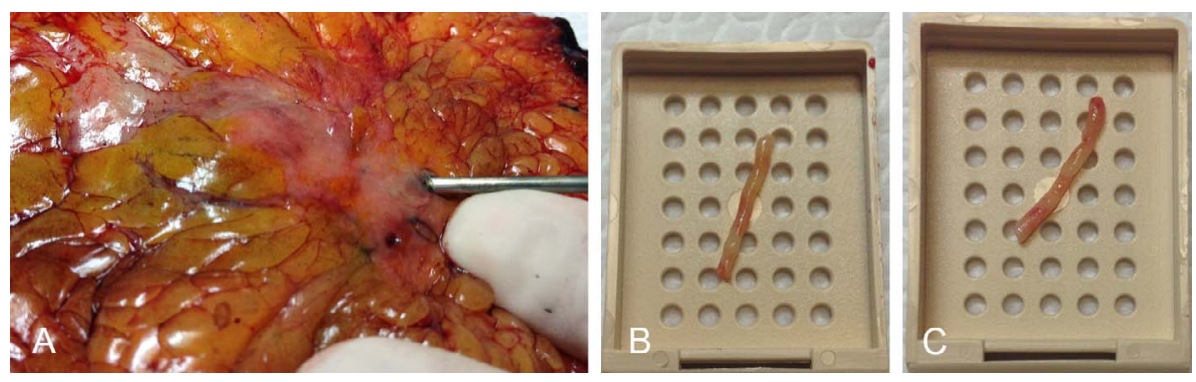

Fig. 9. (A) A resected mastectomy specimen cut in the plane of the tumour. The needle is inserted to obtain a sample. (B) and (C) Examples of obtained samples from a resected specimen. 
Table 1

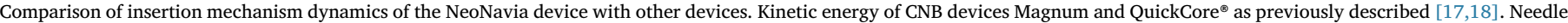
mass includes all accelerated parts. All measurements were performed in air.

\begin{tabular}{|c|c|c|c|}
\hline Name & Quick-Core & Magnum & NeoNavia \\
\hline Device Type & Single-use CNB device & Reusable CNB device with single-use needles & Single-use device \\
\hline Placement mechanism & Mechanical spring & Mechanical spring & Pneumatic driver \\
\hline Control & Manual loading and manual spring release & Manual loading and manual spring release & Automatic \\
\hline Stroke length $[\mathrm{mm}]$ & 20 & 22 & 2.5 \\
\hline Stroke frequency & Single-shot & Single-shot & Multiple consecutive pulses, max. 3 per second \\
\hline Accelerated Needle mass $[\mathrm{g}]$ & 3 & 11 & 3.4 \\
\hline Max. velocity $[\mathrm{m} / \mathrm{s}]$ & 15.59 & 8.19 & 21.2 \\
\hline Max. kinetic Energy [Ws] & 0.36 & 0.37 & 0.76 \\
\hline Needle progression time $[\mathrm{ms}]$ & - & 4.3 & 0.31 \\
\hline Average acceleration $\left[\mathrm{m} / \mathrm{s}^{2}\right]$ & - & 1905 & 480659 \\
\hline Power $[\mathrm{kW}]$ & - & 0.09 & 16.87 \\
\hline
\end{tabular}

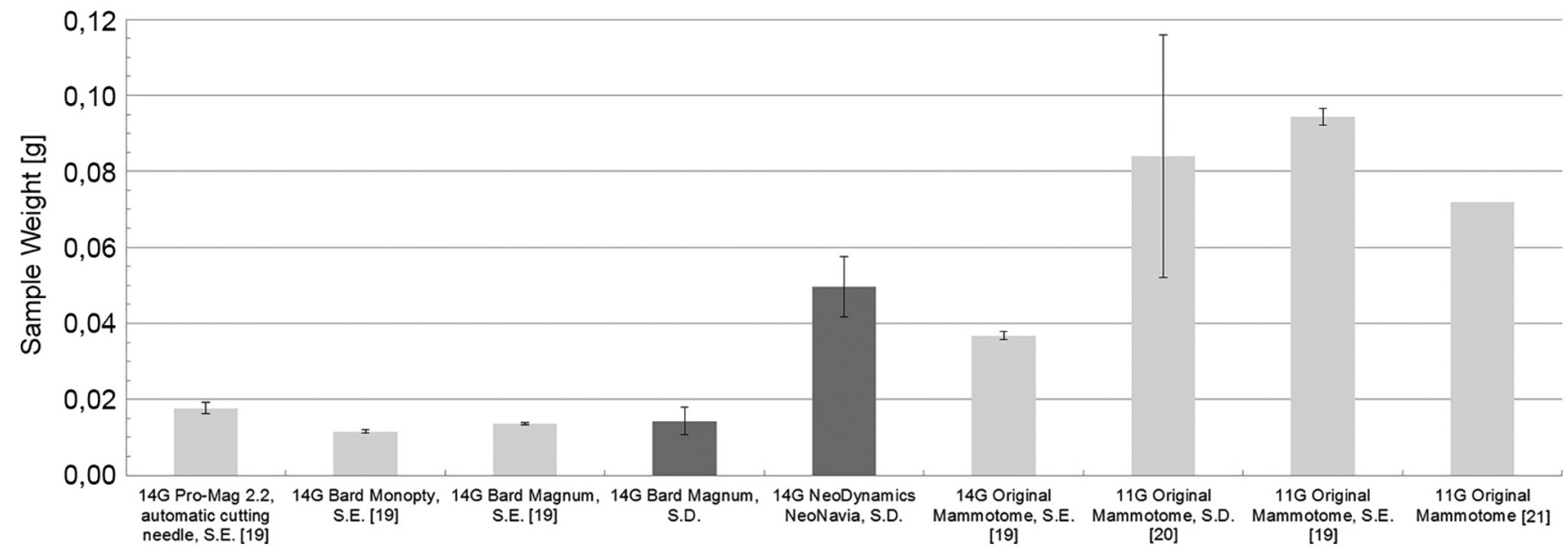

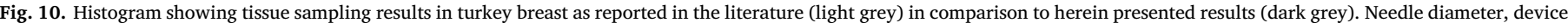
name and reported standard errors (SE) or standard deviations (SD) are given. (See above-mentioned references for further information.)

methodologies. In the longer term, the device could possibly be utilized in other indications such as the liver, prostate, thyroid or kidney.

\section{Acknowledgements}

This work was supported by Sweden's innovation agency (VINNOVA) with grants nr. 2012-00405 and 2013-03508 as well as NeoDynamics AB. The funders had no role in analysis and interpretation of data, writing of the report and decision to submit the article for publication. We kindly thank Magnus Olsen (NeoDynamics), Lars-Peter Svanberg (Etteplan) and Georg Sundström (Sanmina) for their technical expertise as well as Tim Hardman for medical writing support.

\section{Conflict of interest}

The Ph.D. position of Kai-Uwe Schässburger is funded by NeoDynamics $\mathrm{AB}$ and he is a minor shareholder of the company. Stefan Paepke is a consultant to the company.

\section{References}

[1] Silverstein M. Where's the outrage? J Am Coll Surg 2009;208:78-9.

[2] Senkus E, Kyriakides S, Ohno S, Penault-Llorca F, Poortmans P, et al. Primary breast cancer: ESMO Clinical Practice Guidelines for diagnosis, treatment and follow-up. Ann Oncol 2015;26 Suppl 5:v8-30.

[3] Paepke S, Klein E, Metz S, Dittmer S, Schmidt-Feuerheerd N. Aktuelle Aspekte der minimal-invsiven Diagnostik und rekonstruktiven Mammachiurgie [GERMAN]. Journa Onkologie 2016. 04/2016.

[4] Dahabreh IJ, Wieland LS, Adam GP, Halladay C, Lau J, Trikalinos TA. Core needle and open surgical biopsy for diagnosis of breast lesions: an update to the 2009 report. 2014.

[5] Gutwein LG, Ang DN, Liu H, Marshall JK, Hochwald SN, Copeland EM, et al. Utilization of minimally invasive breast biopsy for the evaluation of suspicious breast lesions. Am J Surg 2011;202:127-32.
[6] Raghu M, Hooley R. Breast ultrasound for the interventionalist. Tech Vasc Interv Radiol 2014;17:16-22.

[7] Harvey JA, Moran RE, DeAngelis GA. Technique and pitfalls of ultrasound-guided coreneedle biopsy of the breast. Semin Ultrasound CT MR 2000;21:362-74.

[8] Gruber I, Hahn M, Fehm T, Hann von Weyhern C, Stabler A, Winckelmann A, et al. Relevance and methods of interventional breast sonography in preoperative axillary lymph node staging. Ultraschall Med 2012;33:337-43.

[9] Rjosk-Dendorfer R, Dathe O, de Waal J. Bildgebende und interventionelle Diagnostik [GERMAN]. Manual Mammakarzinom - Empfehlungen zur Diagnostik, Therapie und Nachsorge. 2015. p. 29-60.

[10] Martelotto LG, Ng CK, Piscuoglio S, Weigelt B, Reis-Filho JS. Breast cancer intra-tumor heterogeneity. Breast Cancer Res 2014;16:210.

[11] Kimbung S, Kovacs A, Danielsson A, Bendahl PO, Lovgren K, Frostvik Stolt M, et al Contrasting breast cancer molecular subtypes across serial tumor progression stages: biological and prognostic implications. Oncotarget 2015;6(32):33306-18.

[12] Heverly M, Dupont P, Triedman J. Trajectory optimization for dynamic needle insertion. In: Ieee international conference on robotics and automation (Icra); 2005. p. 1646-51.

[13] Wiksell H, Löfgren L, Schässburger KU, Leifland K, Thorneman K, Auer G. A new method to gently place biopsy needles or treatment electrodes into tissues with high target precision. Phys Med 2016;32(5):724-7. http://dx.doi.org/10.1016/j.ejmp.2016.04.007. [Epub 2016 May 4].

[14] Schässburger K, Svedberg M, Sanell M. Trocar Arrangement for tissue sampling device. International patent publication number WO 2016/058845; 2015.

[15] Moore JZ, McLaughlin PW, Shih AJ. Novel needle cutting edge geometry for end-cut biopsy. Med Phys 2012;39:99-108.

[16] Schässburger K, Sellei P, Lindström B. Distal tip tissue sampling arrangement. International patent publication number WO 2016/058844; 2015.

[17] Wendt O, Siewert C, Luth T, Felix R, Boenick U. Cutting speeds and success of biopsy with different punch biopsy instruments. Radiologe 2001;41:484-90.

[18] Wendt O. Entwicklung einer spulenintegrierten und automatisch gesteuerten Biopsieeinrichtung zur histologischen Abklärung von Kleintumoren in der MRMammadiagnostik [GERMAN]. Medizinischen Fakultät der Charité Universitätsmedizin Berlin; 2004.

[19] Berg WA, Krebs TL, Campassi C, Magder LS, Sun CC. Evaluation of 14- and 11-gauge directional, vacuum-assisted biopsy probes and 14-gauge biopsy guns in a breast parenchymal model. Radiology 1997;205:203-8.

[20] Preibsch H, Baur A, Wietek BM, Kramer B, Staebler A, Claussen CD, et al. Vacuum-assisted breast biopsy with 7-gauge, 8-gauge, 9-gauge, 10-gauge, and 11-gauge needles: how many specimens are necessary? Acta Radiol 2015;56:1078-84.

[21] Poellinger A, Bick U, Freund T, Diekmann S, Hamm B, Diekmann F. Evaluation of 11 gauge and 9-gauge vacuum-assisted breast biopsy systems in a breast parenchymal model. Acad Radiol 2007;14:677-84. 